\title{
BROM'S THREE-PATCH TECHNIQUE FOR REPAIR OF SUPRAVALVULAR AORTIC STENOSIS
}

Mark G. Hazekamp, MD, $\mathrm{PhD}^{\mathrm{a}}$

Arie-Pieter Kappetein, $\mathrm{MD}, \mathrm{PhD}^{\mathrm{a}}$

Paul H. Schoof, MD

Jaap Ottenkamp, MD, $\mathrm{PhD}^{\mathrm{b}}$

Maarten Witsenburg, $\mathrm{MD}, \mathrm{PhD}^{\mathrm{c}}$

Hans A. Huysmans, MD, $\mathrm{PhD}^{\mathrm{a}}$

Ad J. J. C. Bogers, MD, $\mathrm{PhD}^{\mathrm{d}}$
Objective: Case histories of all patients $(n=29)$ operated on for supravalvular aortic stenosis from 1962 to the present were reviewed to study different techniques and outcomes. The technique of symmetric aortoplasty with 3 patches ( 1 in each sinus) is described and compared with other methods. Methods: Case reports were reviewed and follow-up was completed by contacting the patient's (pediatric) cardiologist. We aimed for a last follow-up visit, including Doppler echocardiographic studies, in a period no more than 12 months earlier than December 1997. Supravalvular aortic stenosis was discrete in 25 and diffuse with involvement of the aortic arch and arch vessels in 4 patients. Additional anomalies were bicuspid aortic valve $(n=5)$, coarctation $(n=3)$, ascending aortic aneurysm $(n=1)$, mitral valve insufficiency $(n=2)$, pulmonary valvular stenosis $(n=1)$, and peripheral pulmonary artery stenosis $(n=2)$. Eleven patients had Williams syndrome and 1 patient had Noonan syndrome. Symmetric aortoplasty with 3 patches ( 1 in each sinus) was used in 13 patients, whereas other nonsymmetric methods (1, 2, or Y-shaped patches) were used in 16 patients. Mean follow-up was 10.5 years (range: 4 months-36 years). Results: All techniques adequately decreased the pressure gradient. Progression of preoperative aortic valve insufficiency or de novo regurgitation was not observed except in 1 patient in whom the patches inserted were too large. Conclusions: No difference could be demonstrated in outcome for any surgical technique; however, reconstruction of the aortic root with autologous pericardial patches in each sinus after transection of the aorta has the advantage of symmetry while restoring the normal aortic root anatomy. (J Thorac Cardiovasc Surg 1999;118:252-8)
$S^{4}$ upravalvular aortic stenosis (SVAS) is an uncommon congenital anomaly. SVAS may occur as an isolated form that can be inherited as an autosomal dominant trait, or it may be an important feature of the Williams syndrome. ${ }^{1-3}$ A deletion of the elastin gene results in thickening of the aortic wall characteristically at the sinotubular junction. ${ }^{4}$ The abnormal thickening may extend into the ascending aorta or even into the

From the Departments of Cardiothoracic Surgery ${ }^{\mathrm{a}}$ and Pediatric Cardiology, , Leiden University Medical Centre, Leiden, The Netherlands, and the Departments of Cardiothoracic Surgery ${ }^{\mathrm{d}}$ and Pediatric Cardiology, ${ }^{\mathrm{c}}$ Rotterdam University Hospital, Rotterdam, The Netherlands.

Received for publication Dec 30, 1998; revisions requested March 4, 1999; revisions received April 15, 1999; accepted for publication April 22, 1999.

Address for reprints: Mark G. Hazekamp, MD, PhD, Cardiothoracic Surgeon, Department of Cardiothoracic Surgery, D6-S, Leiden University Medical Centre, PO Box 9600, 2300 RC Leiden, The Netherlands.

Copyright (C) 1999 by Mosby, Inc.

$0022-5223 / 99 \$ 8.00+0 \quad \mathbf{1 2 / 1 / 9 9 6 0 0}$ aortic arch with narrowing of the ascending aorta, aortic arch, and arch vessels. In some patients, pulmonary artery stenosis may be present as well.

Enlargement of the SVAS by means of a single patch is the most commonly used technique to treat the anomaly. ${ }^{5}$ Although the obstruction is adequately relieved by this procedure, the normal configuration of the aortic root and ascending aorta is not restored.

In 1962 our first patient with SVAS was treated by insertion of a single Dacron patch that extended from the ascending aorta into the noncoronary sinus.

An improvement involving reduction of the gradient, as well as restoration of the aortic root, was described by Doty and associates ${ }^{6}$ using an extended aortoplasty involving 2 sinuses instead of only 1 sinus of the aortic root. In our institutions, these techniques (Y- or pantaloon-shaped patches involving 2 sinuses or augmentation of 2 sinuses by 2 separate patches) have been used together with the "single patch extending into to the noncoronary sinus" method.

In search for a better anatomic restoration of SVAS, 
Brom $^{7}$ introduced symmetric aortoplasty with enlargement of all 3 sinuses by the 3-patch technique. The first patient was operated on by this method in 1978. The aorta is transected just distal to the level of maximal obstruction, and 3 pericardial patches are inserted into the aortic sinuses, leading to a normalized geometry of the aortic root with complete relief of the obstruction. ${ }^{7-10}$

In this report our further experience with symmetric aortoplasty with insertion of 3 patches is reviewed and compared with the results of nonsymmetric techniques that we have been using in the past.

\section{Patients and methods}

Patient data. From 1962 to 1996, 29 patients underwent surgical relief of SVAS. Fourteen patients were male and 15 were female. The mean age at operation was $15.8 \pm 12.5$ (SD) years (range, 2.1-46 years). Most patients operated on as adults come from the earlier years of our experience. Williams syndrome was present in 11 patients and Noonan syndrome in 1 patient. Another patient had familial pseudohypoparathyroidism and mental retardation. SVAS was localized in 25 patients and a diffuse form of SVAS was identified in 4 patients. In the diffuse form of SVAS, wall thickening and aortic obstruction extended into the distal ascending aorta, arch, and arch vessels. Three patients with diffuse SVAS had Williams syndrome. Pressure gradients were measured by heart catheterization in all patients. Doppler echocardiographic studies were routinely performed in all with the exception of the patients who were operated on in earlier years. The mean preoperative overall gradient ranged from 40 to $165 \mathrm{~mm} \mathrm{Hg}$ ( $84 \pm 37$ [SD] $\mathrm{mm} \mathrm{Hg}$ ). Aortic valve insufficiency was absent in 24 patients, mild (grade 1/4) in 4, and moderate (grade 2/4) in 1 patient. Left ventricular hypertrophy was evident (electrocardiography, echocardiography) in 28 patients. Restricted access to one of the coronary artery orifices was observed (intraoperatively) in 3 patients. Restriction was due to adherence of the corresponding valve cusp to the sinotubular ridge. Concomitant cardiovascular anomalies were bicuspid aortic valve (5 patients, 17\%), ascending aorta aneurysm (1 patient, $3 \%$ ), aneurysm of the innominate artery (1 patient, $3 \%)$, localized aortic coarctation (3 patients, 10\%), mitral valve insufficiency ( 2 patients, $7 \%$ ), pulmonary valve stenosis (1 patient, $3 \%$ ), and peripheral pulmonary artery stenosis (2 patients, $7 \%$ ).

Surgery. All repairs were performed with cardiopulmonary bypass, moderate hypothermia, and cardioplegic arrest. In earlier years aortic crossclamping with coronary artery perfusion was used. Repair of the aortic arch and arch vessels was performed with the aid of deep hypothermic circulatory arrest in 4 patients with the diffuse form of SVAS. Separate pericardial patches were used for this purpose.

Concomitant procedures were aortic valvotomy in 3 patients and aortic valve replacement in another 6 patients. Indications for valve replacement were stenosis (4 patients) and stenosis/insufficiency ( 2 patients). All 3 patients who underwent aortic valvotomy had bicuspid valves. Two replaced valves were bicuspid and stenotic. One replaced valve was tricuspid and stenotic. The 3 other valves that were replaced all showed cuspal adherence to the sinotubular ridge with restricted access to a coronary orifice. All patients who had an aortic valve replacement together with surgery for discrete SVAS were relatively old $(23,26,33$, and 38 years [2 patients]), with the exception of 1 patient (10 years old). Mitral valve replacement was necessary in 2 patients with serious mitral regurgitation resulting from rupture of the anterior leaflet chorda because of endocarditis in 1 patient and elongation of the anterior leaflet chorda in the second patient. Pulmonary valvotomy was combined with patching of branch pulmonary artery stenosis in 1 patient. Coarctation repair was performed in 3 patients, 2 to 3 weeks after repair of SVAS. One-stage repair would now be preferred.

Three-patch technique. Symmetric aortoplasty was used in 13 patients. The aorta is transected several millimeters distal to the area of maximal stenosis (Fig 1, A). Three longitudinal incisions are made perpendicular to the level of transection. The first incision is usually made into the noncoronary sinus to improve exposure so that the incisions into the 2 coronary sinuses can be safely performed. These incisions are directed into the Valsalva sinuses where they end halfway or deeper if required. The position of the coronary orifices determines the exact location of the incisions into the coronary sinuses. With bicuspid aortic valves the commissural remnant aids in positioning the incisions. The stenotic area is now wide open and ready for restoration (Fig 1, B). Three rectangular patches are inserted (Fig 1,C). Autologous pericardium, untreated or glutaraldehyde tanned (depending on the surgeon's preference), is the material of choice; dura mater and synthetic materials were used in earlier stages of our experience. Patches should not be too wide, because this may lead to commissural stretching and loss of central coaptation with valvular insufficiency as a consequence. The reconstructed aortic root is anastomosed end to end to the distal ascending aorta (Fig 1,D). If deemed necessary, an additional pericardial patch may be used to enlarge the ascending aorta (Fig 1,E).

Nonsymmetric techniques. Nonsymmetric techniques were used in 16 patients, mostly before the technique of symmetric aortoplasty had been introduced. In 14 patients a single patch was inserted after longitudinally opening the ascending aorta across the area of maximal obstruction. Single patches extended into the noncoronary sinus. In 1 patient a Y-shaped patch was inserted into the right and noncoronary sinuses, and in another patient 2 pericardial patches were used (right and noncoronary sinuses) after transection of the aorta.

Follow-up. Follow-up consisted in reviewing all last visits to attending pediatric or adult cardiologists. Follow-up always included Doppler echocardiographic studies. Postoperative heart catheterization was performed in the earlier stages of our experience. We aimed for a last follow-up visit, including Doppler echocardiographic study, in a period no more than 12 months earlier than December 1997. One patient was lost to follow-up because he moved to another country 1 year after the operation, and a second patient could not be followed up for more than 5 years after the operation. In all 


\section{B}
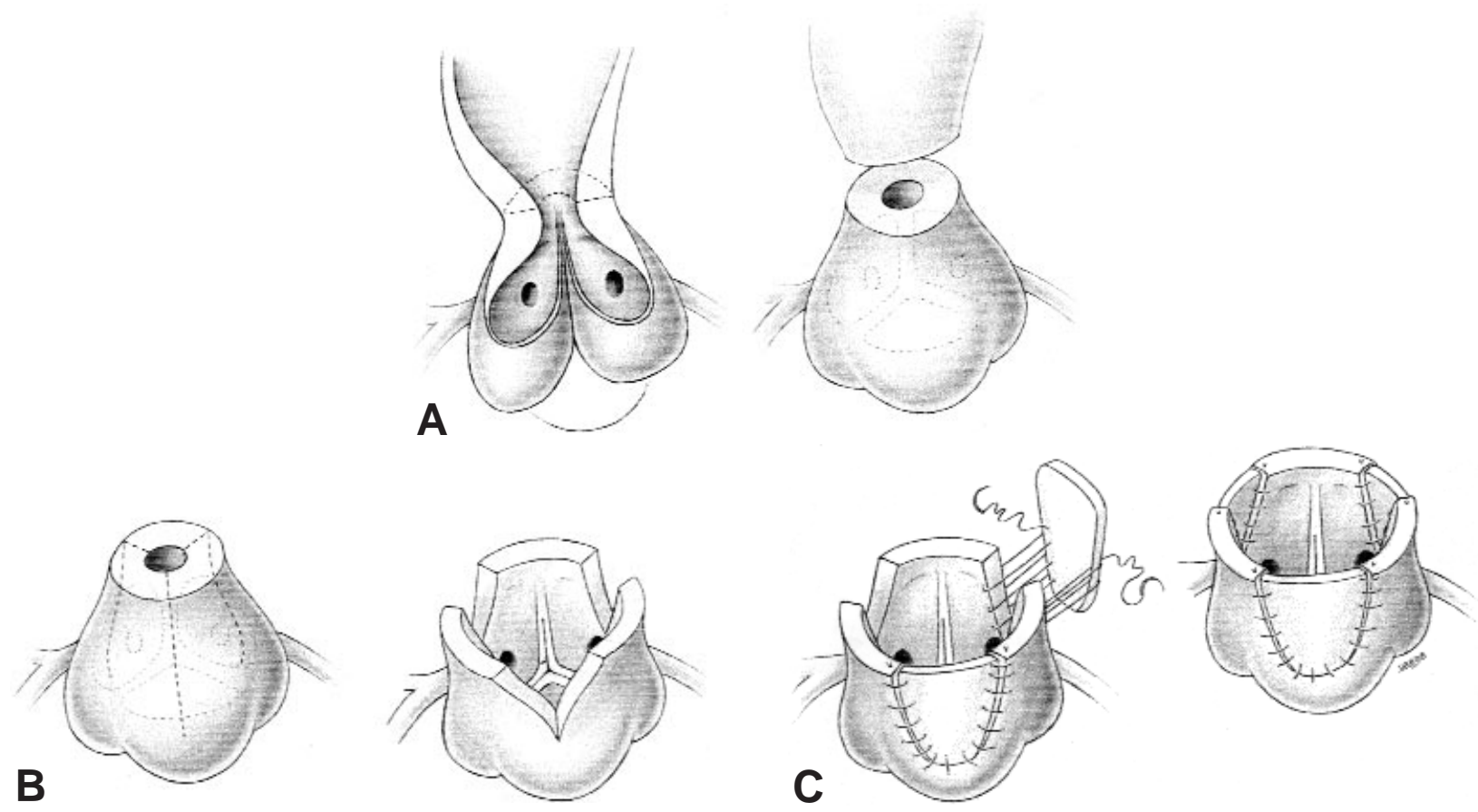

D
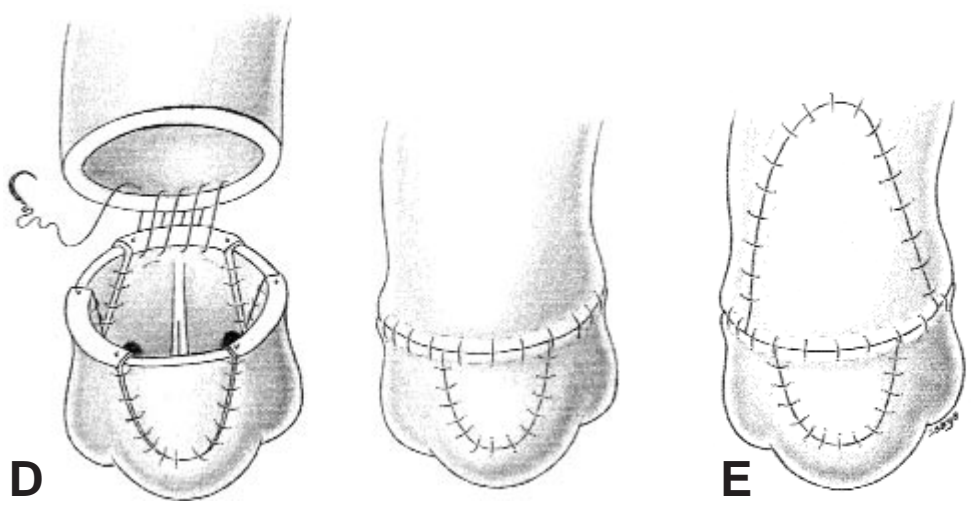

Fig 1. A, The aorta is transected just distal to the area of maximal stenosis. B, Three incisions are made, one into each sinus of Valsalva. C, Restoration of normal aortic root geometry by insertion of 3 patches. D, End-to-end anastomosis of the reconstructed root to the ascending aorta. E, Enlargement of the ascending aorta by an additional patch.

other patients follow-up was complete. Mean length of follow-up was $10.9 \pm 8.1$ (SD) years (range, 0.5-36 years).

All descriptive statistics are expressed as mean \pm standard deviation or mean \pm standard error of the mean. KaplanMeier curves depicting estimations of probability of survival and event-free survival were constructed (Figs 2 and 3). Death and reoperation were considered as events. The logrank test was used to compare survival and event-free survival between the symmetric and asymmetric groups. Group characteristics were compared by means of the Student $t$ test and the $\chi^{2}$ test for quantitative data and categoric variables, respectively.

\section{Results}

Three patients died, 1 early death and 2 late deaths. Postoperative death occurred in a 10-year-old boy operated on in 1965. Severe left ventricular hypertrophy combined with insufficient myocardial protection led to subendocardial infarction and low output failure. One late death occurred in a 50-year-old man who had surgery for SVAS as well as mitral valve replacement 4 years earlier. Notwithstanding a complete relief of the SVAS pressure gradient, left ventricular hypertrophy and ventricular arrhythmias persisted and were pre- 


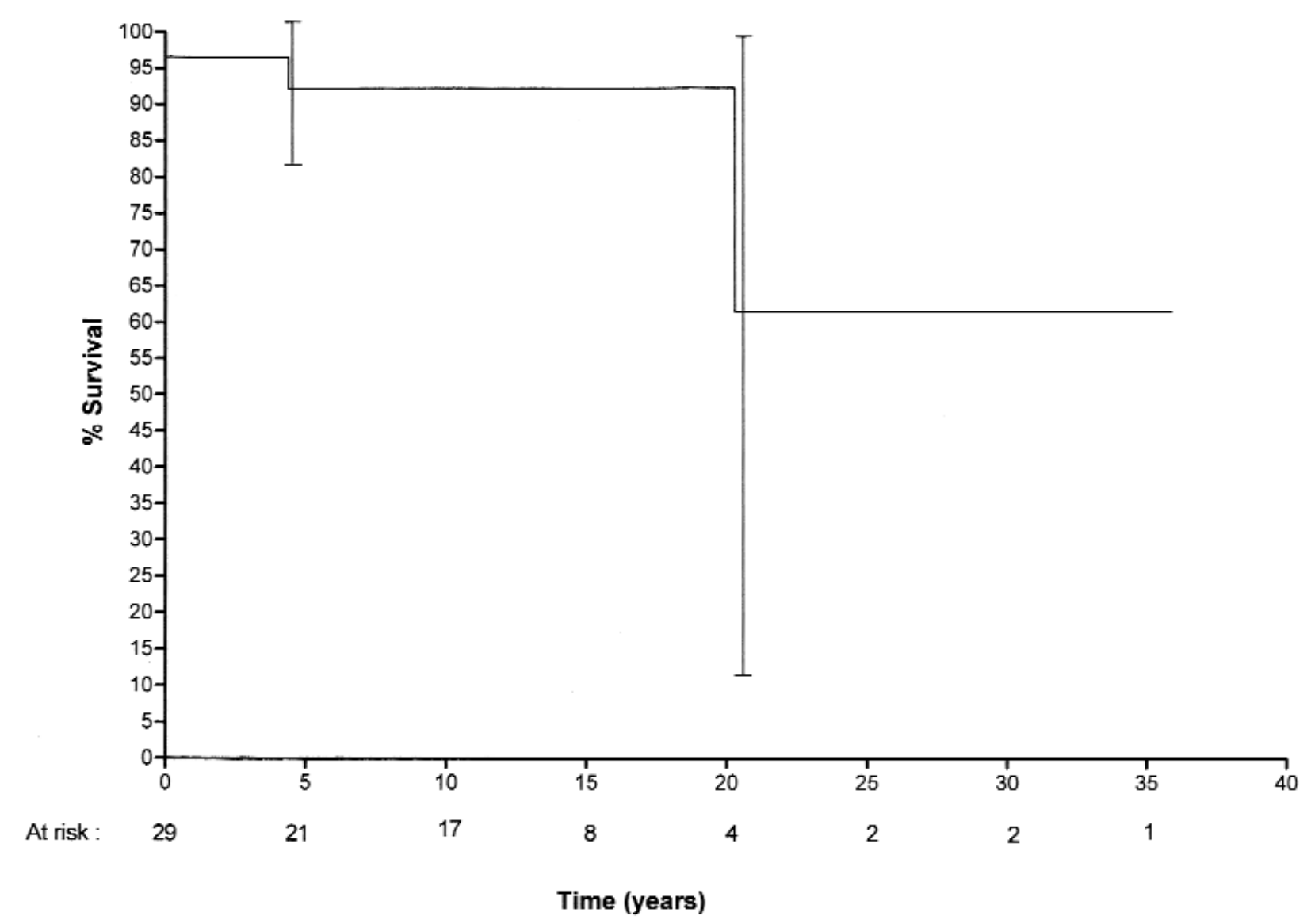

Fig 2. Kaplan-Meier curve of actuarial survival. The number of patients still being followed up is depicted on the horizontal axis at 5-year intervals. The $95 \%$ confidence limits are situated at the time of the events.

sumed to have caused the sudden late death. Another patient died suddenly at home at the age of 40 years. At the age of 19 years SVAS had been relieved by insertion of a single Dacron patch into the noncoronary sinus. Cardiac catheterization 2 years before his death showed absence of any gradient, but echocardiography demonstrated an asymmetric septal hypertrophy.

Actuarial survival at 10 years was $91 \% \pm 5.8 \%$ (SEM) in our patient population (Fig 2). Longest follow-up was 36 years. Survival at 20 years and more is depicted in Fig 2, but because of the small number of patients the survival data may not be reliable (eg, survival at 20 years is $61 \% \pm 25 \%$ ([SEM]). After 1965, no early deaths occurred.

Later aortic valve replacement was performed in 2 patients: in 1 patient 16 years after SVAS repair (single Dacron patch) with aortic valvotomy, and in a second patient 2 years after SVAS surgery. In this latter patient the patches used for symmetric aortoplasty were oversized, resulting in postoperative aortic regurgitation.
In 1 patient with SVAS repair and aortic valve replacement by a Carpentier-Edwards bioprosthesis (Baxter Healthcare Corp, Edwards Division, Santa Ana, Calif), the bioprosthesis was replaced by an aortic homograft 12 years later (Fig 3). In all other patients SVAS repair did not lead to aortic valve insufficiency or progression of a preoperatively existing mild regurgitation. No significant relation between preoperative aortic insufficiency and a higher age at surgery for SVAS could be demonstrated (Student $t$ test, $P=.06$ ).

A significant reduction of mean overall pressure gradients - to less than $10 \mathrm{~mm} \mathrm{Hg}$ - was achieved in all but 4 patients: in these 4 patients the mean postoperative gradients remained 10 to $30 \mathrm{~mm} \mathrm{Hg}$. Of these 4 patients, 2 had a diffuse form of SVAS and 2 other patients had remaining valvular gradients after valvotomy and aortic valve replacement. In 1 other patient bilateral branch pulmonary artery stenosis was not completely relieved after surgical patching. No difference in survival or event-free survival could be demon- 


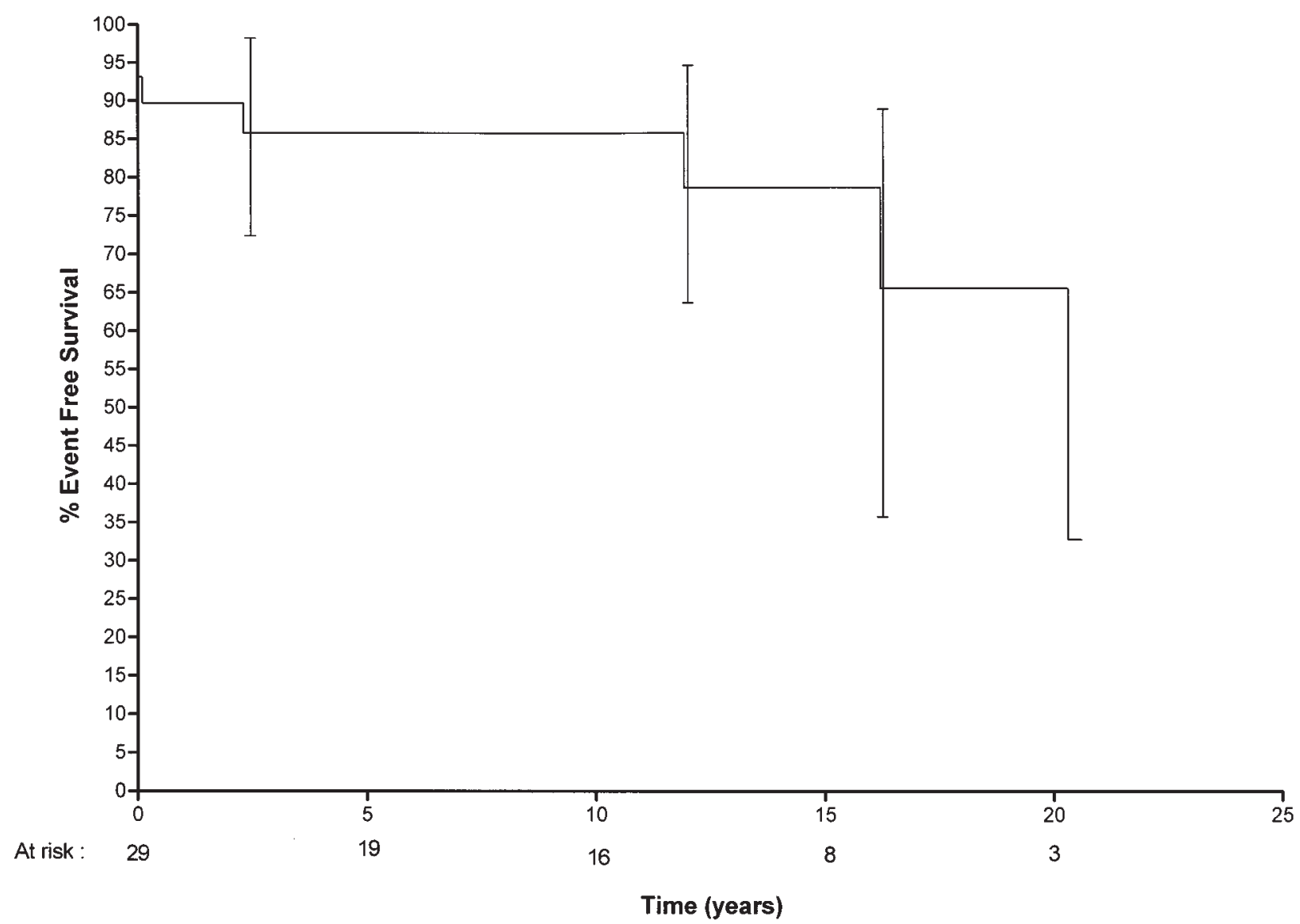

Fig 3. Kaplan-Meier curve of event-free survival. Events are reoperation or death. The number of patients still being followed up is depicted on the horizontal axis at 5-year intervals. The $95 \%$ confidence limits are situated at the time of the events.

strated between the symmetric and nonsymmetric techniques $(P=.83$ and $P=.40$, respectively, log-rank test).

\section{Discussion}

SVAS comprises a spectrum of obstructive lesions that are all characterized by an abnormal thickening of the wall of the major arteries, including the pulmonary arteries. Most commonly, SVAS is a localized obstruction at the level of the sinotubular aortic junction. Other obstructions may however occur in the ascending aorta, the aortic arch, the innominate and carotid arteries, and other major aortic side branches. A localized coarctation is frequently encountered in patients with SVAS. Apart from obstructive lesions caused by arterial wall thickening, other left-sided anomalies include mitral and aortic valve anomalies. Pulmonary valvular stenosis may occur, as well as multiple peripheral pulmonary artery stenosis. ${ }^{11-19}$

In our patient population all these aforementioned anomalies were encountered, as well as 2 aneurysms in the ascending aorta and in the innominate artery. Whether these aneurysms were caused by poststenotic dilatation or by aortic wall degenerative disease remains unclear.

Different techniques are used to repair SVAS. Relief of the stenosis by insertion of a single teardrop-shaped patch into a longitudinal incision that extends across the stenosis from the ascending aorta into the noncoronary sinus was described by McGoon and associates ${ }^{5}$ in 1961. This may be combined with additional resection of the stenotic ridge from within the aorta. Doty, ${ }^{6}$ Delius, ${ }^{13}$ and their associates reported the use of a more extended technique involving the insertion of a Yshaped Dacron patch that branches into the right and the noncoronary sinuses. The incisions are to be positioned approximately $180^{\circ}$ apart. The patch again is inserted across the area of maximal stenosis. The remainder of the stenotic rim above the left coronary sinus may be resected to remove any residual obstruc- 
tion. The technique that has been reported by Myers and associates ${ }^{10}$ obviates the use of prosthetic patch material. The aorta is transected just above the level of stenosis and all thickened tissue is removed. Three vertical incisions are made in the Valsalva sinuses. In the ascending aortic end, 3 longitudinal incisions are made that correspond to the commissures of the aortic valve. With an up-and-down running suture line, the continuity of the aorta is restored, obtaining 3 flaps of aortic tissue that are sewn into the sinuses. Chard and Cartmill $^{21}$ reported a technique that involves resection of the whole stenotic segment, including the more distal parts of the sinuses of Valsalva. The repair is completed by a primary anastomosis of the distal ascending aorta to the aortic root. This method is also symmetric and has no need for prosthetic materials. No long-term follow-up data are available for these newer techniques.

With the exception of the early period (before 1965), hospital mortality did not occur in our group. Nowadays, surgery for SVAS can be safely performed, even if SVAS appears in a more diffuse form. Gradient relief was adequate in all patients with localized forms of SVAS and, with the exception of 1 patient, de novo occurrence or progression of aortic insufficiency did not occur. Surgery in patients with a diffuse form of SVAS is more demanding, with a higher chance of restenosis and residual stenosis. ${ }^{10,14,16,17}$ The presence of aortic valve anomalies such as a bicuspid aortic valve seems to be the most important cause of reoperations in these patients, as has also been stated by others. ${ }^{13,14,16-18}$ Aortic valve replacement may be necessary at the first operation for SVAS or years after initial SVAS surgery. Initial valve replacement is caused by bicuspid valves or adherent leaflets that cannot be repaired. Surgical treatment in infancy may reduce the chance of later aortic valve replacement, especially when the aortic valve is tricuspid and has a normal appearance. Not all aortic valves that had to be replaced were bicuspid, suggesting a negative influence of the long-standing supravalvular obstruction on the aortic valve leaflets. Adherence of 1 valve cusp to the opposite aortic sinus wall was observed in 3 of our patients and resulted in aortic valve replacement in all of them. ${ }^{15,18}$ As has been reported by others, cuspal adherence in 2 of our patients caused paroxysmal obstruction of the left coronary ostium resulting in episodes of syncope and angina. ${ }^{20}$

The localized or "discrete" form of SVAS with maximal obstruction at the sinotubular junction is in reality not a local obstruction but is only one end of the spectrum of obstructive disease of the major arteries. On the basis of a deficiency of the elastin gene, arterial wall thickening may occur in the whole aorta, its major side branches, and even the pulmonary arteries. Furthermore, in a significant number of patients, SVAS appears to form part of a more complex anomaly that not only involves the major arteries but also may harbor abnormalities of the mitral valve, the left ventricular outflow tract, and the aortic valve itself. ${ }^{14}$

As stated by many others, SVAS is a rare disease, and for that reason all reported series are rather small. Because surgical techniques used in most series have differed, ${ }^{10,12-19,21}$ no definite conclusions can be made yet whether long-term outcome of one technique is superior to that of others. In our patient population, long-term outcome demonstrated no difference in outcome between the 3-patch technique and the single, double, or Y-shaped patch techniques, although others have expressed some concern with regard to persisting gradients after single patch aortoplasty. ${ }^{15}$

Nevertheless, we favor the 3-patch technique for treatment of SVAS for several reasons. The 3-patch technique allows for a symmetric reconstruction of the aortic root by enlargement of all sinuses. As has been pointed out by Stamm and associates, ${ }^{18}$ SVAS involves the whole aortic root, and for that reason we consider reconstruction of all 3 sinuses of utmost importance. Anatomic reconstruction of the aortic root is thus combined with a good relief of obstruction. It is important that the patches not be too broad, because this may result in commissural splaying with valvular insufficiency. This mechanism was the presumed cause of aortic insufficiency in 1 of our patients.

Single, double, and Y-shaped patch techniques cannot restore the aortic root in an anatomic fashion. The advancement aortoplasty technique described by Myers and associates, ${ }^{10}$ as well as the 3 -patch technique, results in a better restoration of the aortic root geometry. The use of the advancement aortoplasty may be limited as a consequence of the thickened wall tissue of the ascending aorta. Furthermore, the more diffuse types of SVAS may not be treated by this technique, whereas the 3-patch technique, together with insertion of separate pericardial patches in the ascending aorta and aortic arch, has a better potential for aortic restoration in diffuse SVAS. The technique, as described by Chard and Cartmill, ${ }^{21}$ may bear the same disadvantages as an endto-end anastomosis after excision of a long aortic segment and may cause compression of the left main bronchus or the right pulmonary artery.

\section{REFERENCES}

1. Morrow AG, Waldhausen JA, Peters RL, Bloodwell RD, Braunwald E. Supravalvular aortic stenosis: clinical, hemodynamic and pathologic observations. Circulation 1959;20:1003-10. 
2. Kumar A, Stalker HJ, Williams CA. Concurrence of supravalvular aortic stenosis and peripheral pulmonary stenosis in three generations of a family: a form of arterial dysplasia. Am J Med Genet 1993;45:739-42.

3. Morris CA, Loker J, Ensing G, Stock AD. Supravalvular aortic stenosis consegregates with a familial 6;7 translocation which disrupts the elastin gene. Am J Med Genet 1993;46:737-44.

4. Ewart AK, Jin W, Atkinson D, Morris CA, Keating MT. Supravalvular aortic stenosis associated with a deletion disrupting the elastin gene. J Clin Invest 1994;93:1071-7.

5. McGoon DC, Mankin HT, Vlad P, Kirklin JW. The surgical treatment of supravalvular aortic stenosis. J Thorac Cardiovasc Surg 1961;41:125-33.

6. Doty DB, Polansky DB, Jenson CB. Supravalvular aortic stenosis: repair by extended aortoplasty. J Thorac Cardiovasc Surg 1977;74:362-71.

7. Brom AG. Obstruction of the left ventricular outflow tract. In: Khonsari S, editor. Cardiac surgery: safeguards and pitfalls in operative technique. Rockville (MD); 1988. p. 276-80.

8. Brunelli F, Ghidoni I, Parenzan L. Supraventricular aortic stenosis: a different surgical approach. G Ital Cardiol 1990;20:435-7.

9. Kirklin JW, Barratt-Boyes BG. Congenital aortic stenosis. In: Kirklin JW, Barratt-Boyes BG, editors. Cardiac surgery, 2nd ed. New York: Churchill Livingstone; 1993. p. 1195-237.

10. Myers JL, Waldhausen JA, Cyran SE, Gleason MM, Weber HS, Baylen BG. Results of surgical repair of congenital aortic stenosis. J Thorac Cardiovasc Surg 1993;105:281-7.

11. Becker AE, Becker MJ, Edwards JE. Mitral valvular abnormalities associated with supravalvular aortic stenosis: observations in 3 cases. Am J Cardiol 1972;29:90-4.
12. Braunstein PW Jr, Sade RM, Crawford FA Jr, Oslizlok PC. Repair of supravalvular aortic stenosis: cardiovascular morphometric and hemodynamic results. Ann Thorac Surg 1990;50: 700-7.

13. Delius RE, Steinberg JB, L'Ecuyer T, Doty DB, Behrendt DM. Long-term follow-up of extended aortoplasty for supravalvular aortic stenosis. J Thorac Cardiovasc Surg 1995;109:155-62.

14. Doty DB. Supravalvular aortic stenosis. Ann Thorac Surg 1991; 51:886-7.

15. Flacker G, Teske D, Kilman J, Hosier D, Wooley C. Supravalvular aortic stenosis: a 20 year clinical perspective and experience with patch angioplasty. Am J Cardiol 1983;51:256-60.

16. Sharma BK, Fujiwara H, Hallman GL, Ott DA, Reul GJ, Cooley DA. Supravalvular aortic stenosis: a 29-year review of surgical experience. Ann Thorac Surg 1991;51:1031-9.

17. van Son JA, Danielson GK, Puga FJ, Schaff HV, Rastogi A, Edwards WD, et al. Supravalvular aortic stenosis: long-term results of surgical treatment. J Thorac Cardiovasc Surg 1994; 107:103-14

18. Stamm C, Li J, Ho SY, Redington AN, Anderson RH. The aortic root in supravalvular aortic stenosis: the potential surgical relevance of morphologic findings. J Thorac Cardiovasc Surg 1997;114:16-24.

19. Stewart S, Alexson C, Manning J. Extended aortoplasty to relieve supravalvular aortic stenosis. Ann Thorac Surg 1988;46:427-9.

20. Sun CC, Jacot J, Brenner JI. Sudden death in supravalvular aortic stenosis: fusion of a coronary leaflet to the sinus ridge, dysplasia and stenosis of aortic and pulmonic leaflets. Pediatr Pathol 1992;12:751-9.

21. Chard RB, Cartmill TB. Localized supravalvular aortic stenosis: a new technique for repair. Ann Thorac Surg 1993;55:782-4.

\section{Authoritative}

The Journal of Thoracic and Cardiovascular Surgery is the most frequently cited thoracic/cardiovascular surgery journal in the Science Citation Index. An article in JTCVS is sited on average almost twice as often as those in the closest cardiothoracic journal. 